\title{
Papers
}

\section{Dying of lung cancer or cardiac failure: prospective qualitative interview study of patients and their carers in the community}

\author{
Scott A Murray, Kirsty Boyd, Marilyn Kendall, Allison Worth, T Fred Benton, Hans Clausen
}

\begin{abstract}
Objective To compare the illness trajectories, needs, and service use of patients with cancer and those with advanced non-malignant disease.

Design Qualitative interviews every three months for up to one year with patients, their carers, and key professional carers. Two multidisciplinary focus groups.

Setting Community based.

Participants 20 patients with inoperable lung cancer and 20 patients with advanced cardiac failure and their main informal and professional carers.

Main outcome measures Perspectives of patients and carers about their needs and available services. Results 219 qualitative interviews were carried out. Patients with cardiac failure had a different illness trajectory from the more linear and predictable course of patients with lung cancer. Patients with cardiac failure also had less information about and poorer understanding of their condition and prognosis and were less involved in decision making. The prime concern of patients with lung cancer and their carers was facing death. Frustration, progressive losses, social isolation, and the stress of balancing and monitoring a complex medication regimen dominated the lives of patients with cardiac failure. More health and social services including financial benefits were available to those with lung cancer, although they were not always used effectively. Cardiac patients received less health, social, and palliative care services, and care was often poorly coordinated.

Conclusions Care for people with advanced progressive illnesses is currently prioritised by diagnosis rather than need. End of life care for patients with advanced cardiac failure and other non-malignant diseases should be proactive and designed to meet their specific needs.
\end{abstract}

\section{Introduction}

Improving care for people with advanced illnesses is a national priority. The clinical standards board for Scotland has included palliative care in its generic standards, and the national service framework for coronary heart disease has prioritised advanced disease. ${ }^{1}$
The model of care for cancer patients that encompasses diagnosis, treatment, and palliative care is well developed. ${ }^{23}$ However, patients with non-malignant disease have less access to services including specialist palliative care. Access to services should be based on need not diagnosis. ${ }^{45}$ In the United Kingdom the increasing number of elderly people with advanced non-malignant disease will require palliative care, both generic and specialist. Appropriate and effective models of care that take account of the views of patients and carers need to be developed.

Lung cancer remains the most common and most lethal cancer in Scotland (50\% survival at four months, $20 \%$ at one year). ${ }^{3}$ We compared the experiences of people with lung cancer with those of people with non-malignant disease. We chose cardiac failure as the comparison because it has a higher mortality than many cancers (30\% survival at 18 months) and its prevalence is increasing. ${ }^{6}$

Much research into the end of life has been retrospective, focused on symptom control, psychological distress, or organisational issues and reliant on proxy views rather than the direct patient perspective. ${ }^{7}$ In this longitudinal prospective study we used serial in depth interviews with patients, carers, and key professionals to compare the issues facing patients with lung cancer and end stage cardiac failure and to gauge whether services meet their needs. We used a qualitative research approach to ensure that information could be obtained sensitively from participants with advanced illnesses. ${ }^{8}$

\section{Methods}

Participants

Consultants in respiratory medicine gained outline consent from outpatients with newly diagnosed advanced inoperable lung cancer. Cardiologists and geriatricians identified outpatients with cardiac failure (New York Heart Association grade IV). Before contacting each patient, we telephoned the general practitioner to check if the patient was suitable for recruitment and to seek permission to interview members of the primary care team. Further details of the patients are available from the authors. The samples were chosen purposively to represent the local demography of each condition with respect to age, sex, depri-
Department of
Community Health
Sciences, University
of Edinburgh,
Edinburgh
EH8 9DX
Scott A Murray
senior lecturer in
general practice
Marilyn Kendall
research fellow
Royal Infirmary of
Edinburgh,
Edinburgh
EH3 9YW
Kirsty Boyd
consultant in
palliative medicine
Department of
Nursing Studies,
University of
Edinburgh,
Edinburgh EH8 9JT
Allison Worth
lecturer
St Columba's
Hospice, Edinburgh
EH5 3RW
T Fred Benton
medical director
Western General
Hospital, Edinburgh
EH4 2XU
Hans Clausen
social worker,
palliative care team
Correspondence to:
S A Murray
Scott.Murray@
ed.ac.uk
bmj.com 2002;325:929 
vation category, ${ }^{9}$ living alone or with a carer, and treatment. These variables were based on data from the cancer registry, hospital, and register general and on advice from local specialists.

To recruit 20 patients in each group we approached 84 patients, 59 of whom consented in outline to participate. Further attrition was due to death before the first interview (six), refusal by the carer (two), the patient being too ill or in hospital (four), or the patient not fitting our sampling frame (seven). As no major new themes were emerging after 200 interviews we stopped recruitment.

The study was approved by the local research ethics committee and the primary care and hospital trusts. We obtained written informed consent from study participants and renewed it before each subsequent interview.

\section{Interviews}

MK conducted in depth interviews at three monthly intervals for up to a year with patients and their main informal carer in the patient's home. She asked patients and their carers to talk about the main issues they were facing and their views about the care and support they were receiving. The average age of the 20 patients with lung cancer was 65 years, 15 lived with their spouse, 12 had non-small cell lung cancer, 17 were offered and 16 received chemotherapy or radiotherapy, and five were alive at the end of the study. The average age of the 20 patients with cardiac failure was 74 years, the commonest cause was ischaemic heart disease, 11 lived with a carer, and seven were alive at the end of the study. The average World Health Organization performance status at first interview was 2.0 for lung and 2.6 for cardiac patients, where 0 represents unrestricted activity and 4 represents complete disability. ${ }^{10}$

After each interview we approached the professional carer identified by the patient as being most important to their care. These key informants included general practitioners, hospital doctors, specialist community palliative care nurses, a hospital chaplain, an occupational therapist, a district nurse, a specialist cardiac nurse, hospice doctors, and a warden of sheltered accommodation. At 8-12 weeks after any bereavement we interviewed carers, if appropriate, the general practitioner, and other key professionals. A focus group for each diagnostic group allowed key health and social care professionals, a chaplain, patients, informal carers, and voluntary sector representatives to discuss the issues raised by the interviews and consider alternative service options.

\section{Analysis}

Interviews and focus groups were tape recorded and transcribed (with field notes). Analysis was ongoing throughout the fieldwork to allow emergent themes to be fed back into the data collection. These themes and the research questions formed the basis of the coding strategy. We used the qualitative computer package QSR NVivo and the techniques of narrative analysis. ${ }^{11}$ A second researcher (AW) read all the transcripts and assisted with coding. Regular review and discussion of the evolving themes by the multidisciplinary steering group and data from the focus groups contributed to data synthesis and interpretation.

\section{Results}

The two cohorts yielded 219 interviews: 93 with patients, 53 with carers, and 73 with professionals. We examined the main differences in the illness trajectories, needs, and service use of the two groups (see box 1).

\section{Illness trajectories}

Patients with lung cancer had various initially vague symptoms. Diagnosis was often perceived as delayed, but it was clearly understood once it had been made as was the prognosis. Side effects of treatment made people feel more ill than they had felt before treatment. Many died during or relatively quickly after a course of treatment, though a few had a longer remission.

Cardiac failure was a chronic illness with episodes of acute deterioration that often necessitated emergency admission to an acute hospital, punctuating an overall progressive decline with an unpredictable terminal phase.

\section{Information and understanding of illness and prognosis}

Patients with lung cancer had access to good quality written information and most understood their illness

\section{Box 1: Outline comparison of experience of patients}

\section{Lung cancer}

- Cancer trajectory with clearer terminal phase; able to plan for death

- Initially feel well but told you are ill

- Good understanding of diagnosis and prognosis

- "How long have I got?"

- Relatives anxious

- Swinging between hope and despair

- Lung cancer takes over life and becomes overriding concern

- Treatment calendar dominates life, more contact with services and professionals

- Feel worse on treatment: coping with side effects

- Financial benefits accessible

- Specialist services often available in the community

- Care prioritised early as "cancer" and later as

"terminally ill"

\section{Cardiac failure}

- Gradual decline punctuated by episodes of acute deterioration; sudden, usually unexpected death with no distinct terminal phase

- Feel ill but told you are well

- Little understanding of diagnosis and prognosis

- "I know it won't get better, but I hope it won't get any worse"

- Relatives isolated and exhausted

- Daily grind of hopelessness

- Much comorbidity to cope with; heart often not seen as main issue

- Shrinking social world dominates life, little contact with health and social services

- Feel better on treatment: work of balancing and monitoring in the community

- Less access to benefits with uncertain prognosis

- Specialist services rarely available in the community

- Less priority as a "chronic disease" and less priority later as uncertain if yet "terminally ill" 
and its causes. Many appreciated honesty, although a few patients, carers, and professionals colluded to avoid issues related to dying. Treatment options were discussed but most patients thought they should accept the professionals' recommendations. "As I say, these people are the experts and, you've got to, if you don't abide by what they tell you, its your fault then" (patient 1).

Patients with cardiac failure rarely recalled being given any written information, had a poor understanding of their condition, and, in the absence of chest pain, did not connect symptoms like breathlessness and oedema to their heart. Professionals described complex strategies around giving information, wanting patients to understand their illness but also wanting to protect them from the negative connotations and potential seriousness of their illness implied by cardiac "failure." Prognosis was rarely discussed, and we found little acknowledgment that end stage cardiac failure is a terminal illness. Patients thought about dying in the context of ageing. "I know I won't get better, but I hope it won't get any worse" (patient 2).

Most patients and carers did not feel involved in decision making or empowered to work in partnership with professionals. "I wouldn't say that I take much part in the decision making really. It just happens. They decide and that's that" (patient 3).

\section{Living with illness}

For patients with lung cancer and their carers the prospect of death was a persistent threat. "The doctor told me that you're actually on borrowed time with cancer ... They're hoping to shrink this cancer in the right lung, but you're more or less a time bomb" (patient 4). "You're wondering if you're going to see tomorrow. When I first was told, that was the first thing that went through my head, How long? When? ... it's been like going to hell and back" (patient 5).

Patients worried about how carers would cope and carers worried about upsetting the patient and whether they would know what to do when death did occur. "I feel more for my wife and family. It's strange. You hear people saying that, and I never thought it was true, but you do worry more about what's going to happen to them" (patient 6). "Sometimes I wonder, Is this the end? and then I think, What will it be like, you know? How will I know? And will I know? And what will happen? And I just don't know ... the next time the hospice nurse comes, I'll ask her. So I get myself all braced up to ask her, but when she actually comes, I just can't do it. Perhaps I don't really want to know" (carer of patient 6).

People struggled to maintain a normal life while swinging, often in the same day, from hope to despair. Consequently, people often gave parallel accounts of trying to remain positive while also facing the real possibility of dying. "The main thing is how long I'm going to live. I just keep that to myself. And then I think, just get up girl and show them different; different, determined, positive. And then other times I just sort of weep into it. You can only do so much" (patient 4). "Sometimes I just want to throw the best china at the kitchen wall, but then you just have to grit your teeth and get through it. The only thing is to try and lead an ordinary day" (patient 7).
During chemotherapy or radiotherapy, regular hospital visits and troublesome side effects dominated the lives of both patients and carers. When treatment ended many people felt abandoned and unsupported. "I never knew that cancer would take over your life ... It's like something with a mind of its own, and it tries to control you" (patient 6). "Yesterday I just wanted to curl up in a ball and die, but I know that's not the cancer. That's the treatment. The treatment makes you feel so bad, and each time the after effects have lasted longer and been worse" (patient 7). "I thought there would be something to follow up, even at a later date, once the treatment had finished. But seemingly, I've had all I can get, and now we just have to wait" (patient 8).

Patients with cardiac failure and their carers experienced progressive losses of autonomy and self esteem. All struggled with the daily frustrations of a progressive chronic illness. "You can't do what you did before, things you took for granted are now an impossible dream, I feel useless" (patient 9). "One day I'll be on top, the next day back under again” (patient 10).

Social isolation, comorbidity, and increasing disability were key issues. "I feel like I am in prison in here with him and each day is just like the last" (carer patient 11). “And as I say, the heart problem, didn't equate with us ... because I've got so much else wrong, because the main feature is the arthritis and not being able to move about and being immobile" (patient 12).

Patients and carers had to cope with the variable symptoms and uncertain course of cardiac failure. A major task was the effort of balancing and monitoring complex and frequently changing medication regimens and their side effects in the community. "It's a very fine balance I'm on" (patient 13). "One biggest nuisance is this water tablet, that really limits my movements ... I can't really go anywhere away from the house ... you see, I've got to know where every toilet is wherever I go" (patient 10). "Now your life circulates around trying to keep yourself as good as you can ... You have to try and do as much as you can without harming yourself" (patient 14 ).

Most people had come close to death during acute episodes but, given their many subsequent problems, were left wondering about their present quality of life. "I was sitting in a chair all night ... I would be screaming for air ... very, very frightening ... I suppose it's like drowning really" (patient 12). "It's a life but it's not much of a life. I'm ready for the knacker's yard" (patient 15).

\section{Service provision}

Patients with lung cancer experienced uncertainty and emotional distress while waiting for test results and to start treatment. They lacked support at this time. "Now are they only kidding on they're going to put me on treatment or are they not?" (patient 5).

Most people were satisfied with their care and many appreciated the support of a hospital based lung specialist cancer nurse during treatment. The main practical problem was car parking. Specialist palliative care services offered hospital assessment, community support, day care, and hospice admission. Help with mobility, equipment, and financial benefits was usually available as were carers' support, sitting services, and respite care. Cancer charities and voluntary agencies were actively involved. There was some misunderstand- 
ing among patients, carers, and professionals about the roles of different professionals and agencies, which resulted in failure to access help.

General practitioners sometimes found it hard to judge when to become more actively involved. Most considered it their role to raise psychological, emotional, and spiritual issues but waited for cues, which were not always forthcoming. "But it's not possible with everyone. Some people are very open to it and others are like a brick wall ... You can't make people talk to you about death and dying" (general practitioner of patient 16).

Cardiac patients described poorly coordinated hospital care, lack of continuity, and failure to recognise the involvement and expertise of carers. Primary care contacts were mainly with the general practitioner. There was little planned community support. A few people had developed a long term relationship with a key professional: a consultant, general practitioner, or specialist cardiac nurse. Taking an interest, caring about the person, and good communication skills were valued. Specialist palliative care services were not involved and only a minority had access to a specialist cardiac nurse. Social services, financial benefits advice, carer support, and respite were largely absent, and information and support from cardiac charities were little used. Care was based on a medical model focused on treatment. Lack of services, failure to address end of life issues, and episodes of acute deterioration meant these patients had less opportunity to die at home. "I'm expecting it to be something catastrophic so planning and discussing it isn't really an issue" (general practitioner of patient 17).

General practitioners recognised that there were more resources for patients with cancer and felt frustrated by their own role, which seemed limited to monitoring and adjusting medication. "I'm just a blood leach and monitor" (general practitioner of patient 14). "There's not a lot we can do for people like him" (general practitioner of patient 3).

\section{Discussion}

The contrasting illness trajectories and needs that we have identified point to a model of care for people with cardiac failure that may be different from the cancer model. Many patients with end stage chronic illnesses do not receive appropriate services because their prognosis is uncertain. They could benefit from a dual approach: active management being continued but the possibility of death being acknowledged and discussed. These patients could benefit from the continuity, multidisciplinary care, and focus on symptom control and family support that are the hallmarks of the palliative care approach. ${ }^{512}$ Barriers to extending specialist palliative care services include a lack of appreciation of their role by the non-cancer specialists, lack of expertise of palliative care staff in illnesses other than cancer, a fear of overwhelming workload, and much funding for palliative care services coming from cancer charities. Cardiac nurse specialists, where available, can reduce hospital admissions and improve the coordination of care. ${ }^{6}$ However, adequate psychosocial support is important for everyone who is coping with the social isolation and burden of chronic progressive illness. ${ }^{13} 14$
Suggestions for improving the care of these people are listed in box 2 .

Quality of life improvement teams in the United States have found that comprehensive end of life services are best triggered by the recognition that the patient is "sick enough that dying this year would not be a surprise." If programmes for end of life care targeted those who "reasonably might die," instead of focusing on a prognosis of less than six months, many more patients and their carers would benefit from proactive care. ${ }^{15}$ In primary care, this could include a practice register of such patients, regular patient reviews, identification of key professionals to coordinate care, and more emphasis on multidisciplinary and social support.

Our study confirmed that most people with heart failure do not understand the cause or prognosis of their disease and rarely discuss end of life issues with their professional carers. ${ }^{16-18}$ Unclear prognostic indicators and a desire to protect patients from potentially distressing information are barriers to effective communication between patients and professionals. ${ }^{19}$ The lessons learnt from caring for cancer patients-an individualised approach to information giving, promotion of their coping strategies, appropriate training for professionals-should be applied to those with other life threatening illnesses. ${ }^{20} 21$

\section{Conclusions}

Care for people with advanced progressive illnesses is currently prioritised by diagnosis rather than need. Patients, carers, and professionals perceive the need to address this inequity. End of life care for both groups falls short of the principles of a good death as identified by Age Concern and highlighted by Smith. ${ }^{22}$ Patients with advanced cardiac failure and other non-malignant diseases should receive care that is proactive and designed to meet their specific needs.

We thank all the patients and their informal carers and professional participants for giving their time and energy to this study. We are grateful to staff at Edinburgh Royal Infirmary, the West-

Box 2: Improving end of life care

- All professionals caring for people with an advanced progressive illness should adopt the WHO palliative care approach

- The information needs of patients and carers should be addressed through the use of relevant literature accompanied by individualised explanation

- Strategic planning across primary and secondary care, involving health and social care services working in partnership with specialist palliative care providers, is needed for people with advanced cardiac disease and other progressive illnesses

- Chronic disease management programmes should identify people who "reasonably might die"

- Professionals should receive training in communication skills so that they can discuss diagnosis, prognosis, treatment options, end of life care, and psychological and spiritual issues more effectively with patients and their carers

- Specialist palliative care advice in hospitals and in the community should be available for patients with advanced non-malignant disease

- Palliative care specialists should be trained in the management of advanced non-malignant illnesses 


\section{What is already known on this topic}

The model of care for cancer patients that encompasses diagnosis, treatment, and palliative care is well developed

\section{What this study adds}

Patients with advanced cardiac failure have a different illness trajectory from those with inoperable lung cancer

Such patients and their carers have different concerns, a poorer understanding of the illness and prognosis, and less opportunity to address end of life issues than patients with lung cancer

Health, social, and palliative care services are less readily available to those with a non-cancer diagnosis

Care for patients with advanced cardiac failure should be proactive and designed to meet their specific needs

ern General Hospital, Edinburgh, St John's Hospital, Livingston, and Liberton Hospital, Edinburgh, for identifying patients. We especially thank Dr N Uren, Dr R Fergusson, Dr K Swarski, Dr P Cantley, Ms D Borthwick, and Ms M Smith for help with developing the recruitment strategy. We thank Dr M Dlugoleska of Lothian Health for help with study design.

Contributors: SAM designed the study, oversaw identification of participants, contributed to the interpretation of the data, wrote the paper, and is guarantor. KB helped to design the study, contributed to the interpretation of the data, and wrote the paper. MK recruited the participants, undertook all the interviews, coordinated the data collection, undertook the data analysis, and wrote the paper. AW helped to design the study, read all the transcripts and undertook the analysis with MK, and contributed to writing the paper. TFB helped to design the study, assisted in the focus groups, and contributed to the writing of the paper. HC helped with recruitment and data interpretation and contributed to the writing of the paper.
Funding: Chief Scientist Office of the Scottish Executive.

Competing interests: None declared.

Department of Health. National service framework for coronary heart disease London: Department of Health, 2000.

2 Department of Health. The NHS cancer plan: a plan for investment, a plan for reform. London: Stationery Office, 2000.

3 Scottish Executive Health Department. Cancer scenarios: an aid to planning cancer services in Scotland in the next decade. Edinburgh: Scottish Executive Health Department, 2001

4 Addington-Hall JM. Reaching out: specialist palliative care for adults with non-malignant diseases. London: National Council for Hospices and Specialist Palliative Care Services and Scottish Partnership Agency, 1998.

5 Higginson I, Addington-Hall JM. Palliative care for non-cancer patients. Oxford: Oxford University Press, 2001.

6 Stewart S, Blue L. Improving outcomes in chronic heart failure. London: BMJ Publishing, 2001.

7 Addington-Hall J, McCarthy M. Regional study of care of the dying: methods and sample characteristics. Palliat Med 1995;9:27-35.

8 Green J, Britten N. Qualitative research and evidence based medicine. BMJ 1998;316:1230-3.

9 Carstairs V, Morris R. Deprivation and health in Scotland. Aberdeen: Aberdeen University Press, 1991.

10 WHO. WHO handbook for representing results of cancer treatment. Geneva: WHO, 1979

11 Coffey A, Atkinson P. Making sense of qualitative data: complementary research strategies. London: Sage, 1996.

12 Chavannes N. A palliative approach for COPD and heart failure. Eur J Palliat Care 2001;8:225-7.

13 Kurti LG, O'Dowd T. Dying of non-malignant diseases in general practice.J Palliat Care 1995;11:25-31.

14 Rideout E, Montemuro M. Hope, morale and adaptation in patients with chronic heart failure. J Adv Nurs 1986;11:429-38.

15 Lynn J. Serving patients who may die soon and their families. JAMA 2001;285:925-32.

16 Rogers AE, Addington-Hall JM, Abery AJ, McCoy ASM, Bulpitt C, Coats AJS, et al. Knowledge and communication difficulties for patients with chronic heart failure: qualitative study. BMJ 2000;321:605-7.

17 Lynn J. Learning to care for people with chronic illness facing the end of life. JAMA 2000;284:2508-11.

18 McCarthy M, Addington-Hall J, Ley M. Communication and choice in dying from heart disease. JR Soc Med 1997;90:128-31.

19 The A-M, Hak T, Koeter G, Wal Gvd. Collusion in doctor-patient communication about imminent death: an ethnographic study. $B M$ J 2000;321:1376-81

20 Girgis A, Sanson-Fisher RW. Breaking bad news: consensus guidelines for medical practitioners. J Clin Oncol 1995;13:2449-56.

21 Fallowfield L, Jenkins V, Farewell V, Saul J, Duffy A, Eves R. Efficacy of a cancer research UK communication skills training model for oncologists: a randomised, controlled trial. Lancet 2002;359:650-6.

22 Age Concern. The future of health and care of older people: the best is yet to come. London: Age Concern, 1999.

23 Smith R. A good death. An important aim for health services and for us all. BMJ 2000;320:129-30.

(Accepted 27 August 2002) 